\title{
REFLEXIONES EN TORNO AL RAZONAMIENTO BIOÉTICO EN CUIDADOS PALIATIVOS
}

\author{
REFLECTIONS ABOUT BIOETHICAL REASONING IN PALLIATIVE \\ CARE
}

Constanza Briceño R. ${ }^{1}$

\section{RESUMEN}

Durante las últimas décadas se han observado importantes cambios en materia de salud, incluyendo el aumento de especializaciones, el perfeccionamiento en el control de enfermedades infecciosas, el descubrimiento y desarrollo de nuevos métodos de intervención y el creciente énfasis en la rehabilitación. Así mismo, esto ha favorecido el aumento en la edad promedio de las personas, lo que se ha asociado a una mayor prevalencia de enfermedades crónicas no transmisibles y/o degenerativas. En respuesta a esto, ha sido necesario actualizar la visión y los conocimientos relacionados con los cuidados paliativos, lo que ha significado además, la apertura a un nuevo y complejo campo de acción por parte de los terapeutas ocupacionales. El propósito de este ensayo es realizar una reflexión en torno al razonamiento bioético que como profesionales de la salud debemos llevar a cabo al encontrarnos con usuarios que enfrentan esta situación, recordando que la toma de decisiones éticas es parte de nuestro desempeño cotidiano. De esta manera, en el siguiente texto, se examinarán aspectos considerados fundamentales dentro de la intervención de terapia ocupacional en cuidados paliativos, relacionados con i) la calidad de vida como componente central para cualquier curso de acción a seguir, ii) la facilitación de la ocupación y participación de los usuarios asumiendo el valor ético de éstas (basadas en los principios bioéticos y las éticas del cuidado y de las virtudes), y iii) la dignidad de la persona, promoviendo además, iv) la ética del buen morir como una manera de enfrentar este proceso.

\section{Palabras Clave}

Cuidados Paliativos, razonamiento bioético, calidad de vida.

\footnotetext{
${ }^{1}$ Terapeuta Ocupacional, Licenciada en Ciencia de la Ocupación, Universidad de Chile. Servicio de Medicina Física y Rehabilitación - Servicio de Geriatría, Hospital Clínico Universidad de Chile. Teléfono: 56-9-94997156; Correo electrónico: constanza.briceno.ribot@ gmail.com
} 


\begin{abstract}
In the last decades there have been observed important changes regarding health issues, including the increase of specializations, the perfection of infectious disease control, the discovery and development of novel intervention methods and the growing emphasis on rehabilitation. Likewise, this has favored the increase in the average age of the population, which has been associated with a major prevalence of not transmissible chronic and/or degenerative diseases. As a response to the above, the scope and research related to palliative care has been updated, which has also lead to a broadening of horizons to a new and complex field of action on behalf of occupational therapists. The objective of the present essay is to generate a reflection regarding the bioethical reasoning that we, as health professionals, must keep in mind when finding users that are in said situation, taking in account that ethical decisions are part of our daily performance. Thus, aspects considered to be fundamental within the intervention of occupational therapy in palliative care are to be examined, related to i) quality of life as a central component for any course of action to be applied, ii) the facilitation of the user's occupation and participation, assuming the ethical values of the same (and basing ourselves on the bioethical principles, care ethics and virtues), and iii) the persons dignity, further promoting, iv) the ethics related to a worthy death as a way to face said process.
\end{abstract}

\title{
Key Words
}

Palliative Care, Bioethical Reasoning, quality of life. 


\section{INTRODUCCIÓN}

El concepto de cuidados paliativos es un término relativamente nuevo pero de significativa importancia considerando que alrededor del $60 \%$ de las muertes prematuras a nivel mundial se producen a causa de enfermedades crónicas, principalmente enfermedades cardiovasculares y oncológicas. Es en Inglaterra, alrededor de los años 60, donde se origina un movimiento influenciado por la reacción de personas con enfermedades graves incurables y sus familias con el objetivo de mejorar el apoyo otorgado a esas personas en fase terminal: el movimiento "Hospice" (1). No obstante, es en 1980 cuando se incorpora oficialmente el concepto de cuidados paliativos a la salud mundial. En el año 2002, la Organización Mundial de la Salud (OMS) definió este concepto como "el cuidado activo e integral de pacientes cuya enfermedad no responde a terapéuticas curativas. Su fundamento es el alivio del dolor y otros síntomas acompañantes y la consideración de los problemas psicológicos, sociales y espirituales. El objetivo es alcanzar la máxima calidad de vida posible para el paciente y su familia”. Comúnmente los cuidados paliativos se asocian a enfermedades oncológicas; sin embargo, esta filosofía se aplica a cualquier persona que enfrenta una enfermedad terminal. Según la OMS (1994), los criterios que definen a un individuo con enfermedad en fase avanzada/terminal corresponden a: i) enfermedad incurable, avanzada y progresiva, ii) pronóstico de vida limitado, iii) escasa posibilidad de respuesta a tratamientos específicos, iv) presencia de múltiples síntomas, multifactoriales y cambiantes, v) alto impacto emocional en la unidad paciente-familia, y vi) alta demanda y uso de recursos (2).

En Chile, en los primeros años de la década del 90 y como iniciativa de algunos equipos médicos, se inicia el desarrollo de los cuidados paliativos estableciéndose las bases de un Programa Nacional de Cáncer formulado por el Ministerio de Salud. Tal fue el impacto de este programa, que desde el año 2003 se incorporan los cuidados paliativos (específicamente oncológicos) a las Garantías Explícitas en Salud (GES) visibles en la guía clínica de "Alivio del dolor por cáncer avanzado y cuidados paliativos" (3), lo que permitió ampliar la red de estos cuidados a nivel nacional.

La reflexión en torno a este tema surge a partir de una experiencia personal en un centro de práctica profesional durante el último año de estudio. En aquel contexto se presenta la oportunidad de trabajar con un usuario que llega al servicio de terapia ocupacional con la indicación de cuidados paliativos a causa de un tumor maligno originado en el epitelio de los conductos biliares intra y extrahepáticos, el cual posee una baja probabilidad de sobrevida. Un importante antecedente es que el tumor fue descubierto a partir de la hospitalización del usuario a causa de un accidente cerebro vascular isquémico. En aquel momento, considerando los antecedentes personales y ocupacionales del usuario, así como también, el rápido progreso funcional observado durante el proceso de rehabilitación, la intervención se orientó principalmente a la recuperación de la independencia física y funcional, abarcando en menor grado, los cuidados paliativos y cómo estos impactan en la calidad de vida de la persona. Realizando una reflexión posterior sobre el proceso de toma de decisiones en este caso, es posible visualizar la preferencia de una línea de intervención 
orientada a la funcionalidad, respondiendo a inquietudes y necesidades de la familia y del equipo, dejando de lado la motivación del usuario, quien no se encontraba en completo conocimiento de su situación de salud. De esta forma, surgió la inquietud por comprender la importancia de un adecuado razonamiento clínico previo a cualquier intervención que realicemos durante nuestro quehacer diario, considerando además, que la toma de decisiones éticas es parte del desempeño cotidiano de cualquier profesión del área de la salud, y en este caso, de la terapia ocupacional.

El sustrato reflexivo de la bioética corresponde a actos humanos que afectan o influyen radicalmente en los distintos procesos que vive la persona, por lo que cada acción realizada dentro de nuestra intervención debe encontrarse respaldada y fundamentada por un adecuado razonamiento clínico.

\section{DESARROLLO TEMÁTICO}

\section{Introducción al razonamiento clínico}

Bien es sabido que el razonamiento clínico es la base de nuestra práctica, ya que consiste en el proceso de reflexión y análisis metacognitivo sobre las necesidades de la persona. Es un proceso complejo de diálogo interno que se encuentra implícito en nuestro quehacer profesional, participando en él los fundamentos filosóficos y teóricos que sustentan a la terapia ocupacional y los sistemas de valores y principios éticos, para así entender las necesidades del usuario y tomar decisiones acerca de cómo se debe intervenir (4), evitando posibles riesgos en la salud de la persona y favoreciendo su participación. A partir de este razonamiento se pretende unir y complementar el dominio cognitivo y teórico junto a la capacidad de situarse de manera empática que ha desarrollado el profesional.

Existen diferentes tipos o estrategias de razonamiento clínico, descritos por Mattingly y Fleming en Clinical reasoning: Forms of inquiry in a therapeutic practice (5):

- De procedimiento: existe un desarrollo lógico que comienza con la identificación del problema y las necesidades de la persona, a modo de desarrollar un plan basado en un marco de referencia. Este tipo de razonamiento se basa en los factores del usuario, sus funciones y estructuras corporales.

- Interactivo: permite desarrollar una comprensión de la persona y su familia para seleccionar y adaptar la intervención que privilegie el interés y atención del usuario. Busca generar una comprensión del sujeto y su familia como individuos, sin basarse únicamente en el diagnóstico.

- Condicional: visualiza de manera integral (usuario, situación de salud, familia y medio) el actual momento, considerando las posibilidades de cambio. Se evalúa el compromiso y posible participación del usuario para realizar una selección de actividades significativas para la persona, promoviendo así, un sentido de propósito y creando intencionalidad. 
- Intuitivo: busca comprender las preferencias e intereses del usuario para diseñar las actividades de intervención. Se interpretan claves interactivas para ofrecer compromiso y participación, relacionando las actividades seleccionadas con las metas de intervención e intereses de la persona (4).

Estas estrategias de razonamiento clínico, en la práctica, se encuentran entrelazados por distintos aspectos o enfoques de pensamiento de acuerdo a la naturaleza de la situación de salud que se está tratando, o bien, según las diversas demandas de los problemas a tratar. Éstas corresponderían al razonamiento científico, el cual permite vislumbrar el problema de salud, su intensidad y como éste influye en el desempeño ocupacional de la persona (¿cuál es la naturaleza de la enfermedad?, ¿qué protocolos de intervención son aplicables a su problema de salud?); narrativo, el que permite comprender el significado del problema para el usuario desde su propia narrativa (¿qué ocupaciones son más significativas para esta persona?); pragmático, relacionado al contexto de la práctica y del usuario (¿qué recursos de la familia o de personas a cargo existen para apoyar la intervención?); y ético, el cual permite elegir acciones moralmente defendibles (¿cuáles son los beneficios y riesgos para la persona relacionados con la intervención?). Es en este último punto en el cual se basa este ensayo, ya que el proceso de razonamiento clínico termina en una decisión ética por sobre una científica, siendo la naturaleza ética de este razonamiento por la cual se debe guiar toda la secuencia de intervención a realizar (6).

\section{Bioética y razonamiento bioético}

En vez de hablar de razonamiento ético, en este ensayo se hablará de razonamiento bioético, entendiendo a esto último como el estudio sistemático de la conducta humana en el área de las ciencias de la vida y la atención de la salud, en cuanto dicha conducta es examinada a la luz de los principios y valores morales (7). Una de las principales herramientas que utiliza la bioética es el diálogo, el cual alude a la participación en prácticas sociales discursivas o deliberativas, siendo ésta la manera en la que se puede generar un clima social, psicológico e intelectual en el que pueden producirse decisiones adecuadas y prudentes. En todo ámbito de toma de decisiones es fundamental el diálogo; no obstante, para la terapia ocupacional adquiere especial relevancia, ya que nuestro quehacer está centrado i) en la persona, manteniendo el compromiso de considerar al sujeto como un agente activo dentro de su propio proceso de rehabilitación, ii) en la ocupación de esta persona, considerando aquellas actividades que tengan propósito, sentido y significado para ésta, y iii) en la evidencia, ya que la búsqueda e implementación de intervenciones basadas en este último punto se convierte en una actitud ética por si misma. Para esto se requiere el diálogo, al ser la discusión el instrumento básico para la toma de decisiones, la cual debe realizarse entre el profesional o equipo interdisciplinario, junto al usuario y/o su familia o cuidador. Así, el diálogo es una de las herramientas más importantes que el discurso bioético ha aportado a nuestra profesión (8).

La bioética posee distintos métodos de reflexión, entre los que se encuentran la casuística, la narrativa y el principialismo (7). Según Lolas, la casuística se refiere "a identificar ejemplos y analogías, apelar al conocimiento intuitivo almacenado en máximas y aforismos, analizar las circunstancias, considerar grados de probabilidad en la comisión u 
omisión de ciertos actos, emplear argumentos acumulativos y resumir la experiencia en un juicio final", es decir, considerar la singularidad de cada sujeto en cada caso particular. Por otro lado, la narrativa puede ser considerada como la forma de incorporar lo histórico al estudio del comportamiento moral, y al igual que la casuística, apela a la razón discursiva. No obstante, es el principialismo el método ampliamente utilizado por los profesionales del área de la salud, y particularmente por los terapeutas ocupacionales. Esta metodología postula la existencia de cuatro principios que servirían de marco para el análisis de los problemas que pueden plantearse a partir de la práctica de las distintas ciencias de la vida. Estos principios corresponden al de no-maleficencia, el cual establece que no se debe causar daño a otra persona; y el de justicia, el cual supone el trato igualitario y la imparcialidad en la distribución de cuidados y recursos. Ambos principios constituyen el nivel de mínimos morales, es decir, determinan el comportamiento que protege la dignidad humana. Por otro lado, se encuentra el principio de beneficencia, entendido como la obligación de beneficiar o hacer el bien; y el de autonomía, relacionado con la capacidad de autodeterminación del individuo, es decir, hacer lo que realmente la persona desea hacer, incluyendo la aceptación de las prioridades y objetivos del usuario, sin ocultar información solicitada por el mismo $(9,10)$. Ambos constituyen el nivel de máximos morales, determinando lo que cada persona elige libremente hacer, explicitando los actos de índole subjetivo, dentro de la esfera privada del individuo.

Incluso, estos principios se encuentran implícitos en los distintos códigos éticos, internacionales y nacionales, que sustentan nuestra profesión, como por ejemplo, en el Código de Ética de Terapia Ocupacional aprobado por la $\mathrm{AOTA}^{2}$, el Código de Ética de la WFOT $^{3}$, las Normas de Práctica para el Ejercicio Profesional del $\operatorname{COTEC}^{4}$ (8) y el Código de Ética Profesional del Colegio de Terapeutas Ocupacionales de Chile. Sin embargo, si bien estos principios bioéticos son generales, los problemas que surgen en el ámbito de la salud y participación ocupacional, son particulares y requieren, en consecuencia, de soluciones concretas y ajustadas a cada situación.

\section{Razonamiento bioético, cuidados paliativos y terapia ocupacional}

Para situarnos en el contexto a partir del cual surge el tema de este ensayo es importante entregar una referencia del concepto de cuidados paliativos. La escena de la muerte, que antes ocurría predominantemente en el ambiente familiar, se transfiere y comienza a suceder en el hospital $u$ otras instituciones, lo que implica la ruptura con el entorno habitual de la persona en tal situación. Y si bien durante el último tiempo se ha vuelto a reconocer la importancia de la muerte en un ambiente familiar, en la práctica se puede observar que ésta continúa ocurriendo en instituciones. Pareciera que existen diversos obstáculos que surgen en el momento en que a la familia se le plantea la opción de asumir la responsabilidad de cuidar al usuario en casa, como por ejemplo, razones de índole psicológico, relacionadas a la claudicación emocional de la familia; razones sociológicas, dentro de las que se podrían incluir la estructura de la familia moderna, las diferencias entre el medio rural o urbano, las

\footnotetext{
${ }^{2}$ Asociación Americana de Terapia Ocupacional, actualizado en 2007.

${ }^{3}$ Federación Mundial de Terapeutas Ocupacionales (1992), revisado en 2004.

${ }^{4}$ Comité Europeo de Terapeutas Ocupacionales (1991), actualizado en 1996.
} 
condiciones laborales o la evolución demográfica; o bien, razones de seguridad, de competencia o económicas (11).

Respecto de la esencia del concepto de cuidado paliativo, se destaca el alivio de los síntomas, del dolor y del sufrimiento en los usuarios que sufren de enfermedades crónicodegenerativas o que están en la fase final de una enfermedad, y la búsqueda de la mejora de su calidad de vida. En este punto es importante recalcar que al hablar de dolor en cuidados paliativos se considera la definición planteada por Cecily Saunders, quien propone el concepto de dolor total, refiriéndose a una visión global del sufrimiento, donde se incluyen sus dimensiones física, social, emocional y espiritual (12).

En relación a la terapia ocupacional, el propósito de la intervención es mejorar la calidad de la sobrevida, de modo que el usuario tenga las mejores condiciones posibles de confort y productividad, y que su desempeño tenga lugar con un mínimo nivel de dependencia, cualquiera sea su expectativa de vida (13). Para esto, se deben considerar los principios de atención integral (aspectos físicos, emocionales, sociales y espirituales), tanto al usuario como a su familia, promoviendo la autonomía y dignidad en las decisiones terapéuticas, incluyendo los principios bioéticos en una concepción terapéutica activa. Por tanto, una intervención básica debería incluir el control de síntomas y apoyo emocional al usuario y su familia, manteniendo un adecuado nivel de confianza, respeto, soporte y comunicación. De esta manera, dentro de los objetivos generales de la intervención se encontraría el mejorar la calidad de vida del usuario, integrando los aspectos psicológicos y espirituales en el cuidado, apoyando a la familia tanto durante la enfermedad del usuario como también durante el duelo.

Habitualmente, al tratar con usuarios que padecen de una enfermedad terminal, resulta lógica la plena utilización de una metodología principalista dentro del razonamiento bioético. Sin embargo, ésta por si sola puede ser insuficiente en los cuidados paliativos, y son las éticas del cuidado y de las virtudes las que se presentan como apropiadas y necesarias de incorporar en este ámbito (10). La primera enfatiza la naturaleza vulnerable y dependiente de los seres humanos, destacando no solo el proceso de decisión, sino que también la calidad de las relaciones reflejadas en la continuidad, apertura y confianza. De igual manera, la ética del cuidado está basada en el supuesto de que el cuidado es transformador, ya que el cuidar a alguien involucra el ayudar a que crezca y se actualice, siendo éste un proceso, una forma de relacionarse con otros que implica un desarrollo (14). Por otro lado, la segunda acentúa la importancia de acciones virtuosas en el acercamiento ético al usuario, poniendo el acento en las cualidades del agente moral, encontrándose aludida en el concepto de virtud (15), cualidad humana que permite a quien la posee tomar decisiones apropiadas en situaciones adversas.

\section{Calidad de vida}

En la introducción se menciona la definición de cuidados paliativos propuesta por la OMS, donde se destaca la consideración de otros factores relevantes dentro del bienestar de la persona, como son las dimensiones psicológicas, sociales y espirituales del usuario. No 
obstante, es fundamental incluir la dimensión ocupacional y su relación con la calidad de vida, siendo este concepto mencionado de manera infaltable al hablar de cuidados paliativos.

Podría parecer incompatible incorporar una perspectiva rehabilitadora dentro de lo paliativo, ya que la rehabilitación se suele asociar a la recuperación, mientras que los cuidados paliativos se centran en los últimos momentos de vida. Sin embargo, ambos conceptos comparten objetivos y enfoques terapéuticos comunes, ya que la rehabilitación puede mejorar el cuidado y la satisfacción de las personas que reciben cuidados paliativos. Así, a través de la rehabilitación se buscaría mantener la función y la independencia por el mayor tiempo posible para mejorar la calidad de vida del usuario y reducir la carga de los cuidadores (2).

La mejora de la calidad de vida es un objetivo primordial para la práctica de la terapia ocupacional en todo ámbito, la cual busca ayudar a las personas a realizar actividades que sean importantes para su salud y bienestar a través de la participación en ocupaciones valiosas. De esta manera, el tema de la calidad de vida, desde una perspectiva ocupacional, solo puede plantearse a partir de una posición que de prioridad a la autonomía de la persona y a su derecho a evaluar distintas opciones y eventualmente rechazar intervenciones. Así mismo, al plantearse intervenir en búsqueda de una mejor calidad de vida, se deben considerar los distintos elementos que componen este concepto; es decir, la calidad de vida como concepto subjetivo, multidimensional, complejo y dinámico (16).

\section{Valor ético de la ocupación y participación}

Volviendo al razonamiento bioético es importante incluir dentro de éste una perspectiva ocupacional predominante y cercana a los conceptos básicos de nuestra profesión, recurriendo al valor ético que se le puede asignar a la ocupación y participación de la persona. El ideal del trabajo del terapeuta ocupacional será poder reconocer el desafío de crear distintas oportunidades para la actividad y la participación, independiente de las capacidades que posea la persona o el grado de severidad de su situación terminal. Para esto, se sugiere propiciar actividades significativas para la persona, ya que una actividad, por el solo hecho de ser tal, no es suficiente. De esta manera, se le debe asignar un valor o significado a la actividad, el cual sea coherente con los deseos de la persona, y que además le proporcione un cierto grado de satisfacción al llevarla a cabo, dentro de un nivel de dominio relativo de la actividad. Por otro lado, en palabras de Dige, la motivación resalta como un elemento básico para la participación, la cual implica ser parte, involucrarse, comprometerse y tomar decisiones (17). Todo lo antes mencionado se enmarca dentro de la justicia ocupacional, concepto de gran complejidad que permite reconocer la preocupación por la facilitación en la distribución de bienes y derechos igualitarios, a través de un enfoque sobre las relaciones entre la salud, calidad de vida y ocupaciones con sentido (18). En otras palabras, ¿por qué una persona en situación de enfermedad terminal podría no tener iguales oportunidades de participación en ocupaciones que sean significativas para sí misma? Así, es tarea del terapeuta ocupacional regirse por los principios morales universales al demostrar respeto por las personas, hacer el bien, evitar el daño y ser justo; no obstante, se debe avanzar un paso más allá, y otorgar el valor ético necesario a la ocupación y participación. 


\section{Ética del buen morir}

Es importante recordar que el objetivo de los cuidados paliativos es la calidad de vida, sin alargar la supervivencia ni producir daños innecesarios. De esta manera, dentro de nuestra labor es factible incluir la reflexión del buen morir junto a nuestros usuarios, lo cual, en muchos casos, no se considera. Según Lolas, el tema de la muerte es para muchas personas un tema controversial, ya que es en el plano individual humano donde se conjugan las emociones sobre la ausencia, la desaparición de un orden o estructuras peculiares, el derrumbe de proyectos y expectativas, y las consecuencias sociales y legales de la pérdida de una persona. De allí el debate sobre el proceso de muerte versus el estado de muerte. Este último se supone irreversible, mientras que el primero genera la incertidumbre sobre el curso que tomará (19).

En el proceso de la muerte y el morir se conjugan valores y derechos que la bioética desglosa y atiende, desde la autonomía que respalda la capacidad objetora por motivos de conciencia hasta la dignidad ineludible para decidir ese momento único e irreversible del morir. Es necesario aprender a morir (20) y en esto podemos convertirnos en facilitadores para nuestros usuarios.

\section{Dignidad}

Por otro lado, se destaca la aparición del concepto de dignidad, el cual, según la Declaración Universal sobre Bioética y Derechos Humanos, es ubicado como un referente ético central para la ciencia y tecnología, debiendo ser respetado en su totalidad. La dignidad, es por tanto, fundamental en la práctica de la terapia ocupacional y toma un papel esencial en las intervenciones en cuidados paliativos.

El razonamiento bioético, en estos casos, debe considerar que a partir de una formación individual primordialmente dialógica, de construcción de la identidad a partir del otro, la dignidad aparece como un concepto relacional, ya que ésta se construye en el interior del tejido cultural de las relaciones materiales y simbólicas. Es además, un concepto de gran complejidad cultural y dinamismo histórico, ya que se centra en la protección de los sujetos al contemplar dimensiones humanas como el autogobierno y, más que eso, la responsabilidad moral en relación al otro (21).

Se ha mencionado implícitamente a lo largo del ensayo, que un elemento central en el razonamiento bioético que puede realizar el terapeuta ocupacional en estos casos tiene relación con un aspecto esencial de nuestro quehacer: la práctica centrada en el usuario. Cualquier decisión del usuario es el resultado de su derecho de autonomía o autodeterminación, y del respeto de su dignidad. De esta manera, el profesional además utilizará la ocupación significativa como una herramienta primordial para ayudar a mantener y restaurar el bienestar. La ocupación ha sido propuesta como una fuente primaria de propósito y significado en nuestra vida, pero también, como se ha desarrollado a lo largo de este ensayo, en nuestra muerte. 
La calidad de vida no se trata solamente del control del dolor y de proporcionar confort. Se trata además de mejorar la habilidad para realizar actividades importantes para la persona y su familia, creando oportunidades para vivir plena y productivamente hasta la muerte. De esta manera, en su intervención, el terapeuta ocupacional puede considerar aspectos desde la búsqueda de independencia de las actividades diarias y reestructuración de los patrones de ocupación hasta la toma de decisiones relativas a las metas que el usuario tiene para el final de su vida. Por tanto, en el razonamiento bioético es fundamental considerar las necesidades ocupacionales reales y atingentes a la situación que se encuentra viviendo la persona y entender la ocupación como una experiencia irrepetible y construida personalmente por un individuo. Es decir, la ocupación como un evento subjetivo en condiciones temporales, sociales y culturales que son únicas. De igual manera, dentro del razonamiento bioético deben considerarse los principios básicos de los cuidados paliativos, adoptando un acercamiento holístico que tome en cuenta las experiencias de la vida de la persona y su situación actual, respetando sus derechos fundamentales, dentro de los cuales se incluye el recibir la asistencia en salud necesaria, ser respetado en su dignidad, y ser apoyado y cuidado en sus necesidades. Así mismo, tienen derecho al cuidado del dolor y del sufrimiento, a ser informados, a la autodeterminación y a la suspensión de intervenciones.

La filosofía de los cuidados paliativos se preocupa básicamente de la calidad, valor, significado y sentido de la vida. En este punto es posible distinguir los conceptos de sentido y significado; siendo el primero una percepción interior del propio individuo, decantada en las tradiciones, conceptos, creencias e ideas personales. La propia vida cobra sentido cuando aparece alentada por un propósito, cohesionada por ideales y esperanzas. Por otro lado, en el significado se le da importancia a lo que la sociedad y el entorno valora en relación a lo que personalmente se considera con sentido (22).

\section{Consideraciones finales para el razonamiento bioético en cuidados paliativos}

Es por todo lo anterior que probablemente uno de los mayores desafíos dentro del razonamiento bioético (basado en el diálogo y la responsabilidad) en estos casos, sea otorgar un nuevo sentido a la persona en relación a la muerte, entregando una atención bioéticamente inspirada que integre lo apropiado según el arte, lo bueno según la virtud y lo justo según el uso social, siempre considerando que lo que para nosotros como profesionales puede parecer evidente de realizar, si no contempla la opinión de los propios usuarios y sus familias, se hace impracticable o inútil.

A partir de la revisión bibliográfica realizada es posible presentar una propuesta de razonamiento bioético que sirva de guía para los terapeutas ocupacionales que enfrenten un conflicto en la toma de decisiones en casos de esta índole. Para esto, es importante considerar que se pueden integrar las tres metodologías que propone la bioética para la toma de decisiones. Basándonos en el esquema de la técnica de resolución de problemas sugerido por D’Zurilla y Goldfried (23), en primer lugar, debe realizarse una orientación hacia el problema, que incluya la percepción, atribución y valoración del mismo. En esta etapa se deben considerar los antecedentes del caso, conociendo aspectos como los criterios técnicos, factores económicos involucrados, naturaleza ética del problema, aspectos legales, 
calidad de vida y preferencias de los usuarios. Estos dos últimos elementos se relacionan directamente con lo planteado en párrafos anteriores. Desde la fase inicial del razonamiento bioético debe incorporarse el análisis de la calidad de vida del usuario, del valor ético que se le puede asignar a la ocupación y participación de la persona a través de una futura intervención, y de la incorporación de la ética del buen morir, manteniendo siempre, como prioridad, el respeto por la dignidad de la persona y el cumplimiento de los principios bioéticos. En este último punto toma especial relevancia el principio de autonomía, ya que como terapeutas ocupacionales debemos actuar como facilitadores para ayudar al usuario a ser, en el mayor grado posible y en todo momento (particularmente en aquel cercano a la muerte), el protagonista de su propia vida.

Una vez definido el problema, se debe realizar una propuesta concreta de alternativas que incorporen todos los elementos y factores mencionados anteriormente, para luego continuar con el proceso de toma decisiones y finalmente poner en práctica la solución elegida como apropiada, siempre considerando lo que el usuario define como prioritario. De igual manera, es posible utilizar otras herramientas que faciliten el proceso de toma de decisiones, como por ejemplo, la formulación de un árbol de decisiones, el cual permite elegir una alternativa entre varios cursos de acción propuestos. Lo interesante de esta herramienta es que permite estimar cuáles son las distintas opciones, investigar sobre las posibles consecuencias de cada una y construir una imagen balanceada de los riesgos y beneficios, recordando y otorgando un papel primordial a las consideraciones antes mencionadas.

Si bien se ha mencionado la relevancia de considerar cada caso de manera particular incluyendo las necesidades auto-percibidas por parte de los usuarios, la propuesta recién sugerida responde a la idea de profesionalizarnos frente a este tema, requiriendo indispensablemente investigar y reflexionar en torno a los cuidados paliativos desde nuestra área.

\section{CONCLUSIONES}

En materia de salud ha habido diversos cambios durante el último siglo que se han manifestado en importantes cambios socio-demográficos, como lo es el envejecimiento de la población, con todos los desafíos que esto conlleva. Uno de ellos se encuentra asociado al aumento de la edad promedio de los usuarios, ya que existe una mayor prevalencia de enfermedades crónicas y/o degenerativas. En respuesta a esto, se han actualizado la visión y los conocimientos relacionados con los cuidados paliativos, y se ha adoptado una aproximación activa de estos cuidados. En este último punto se destaca la labor de la terapia ocupacional, que a través de la facilitación de la ocupación y participación, ha aportado una perspectiva distinta a la intervención en estos casos. Sin embargo, previo a cualquier acción, es fundamental desarrollar un razonamiento bioético que valide y se ajuste a los intereses y deseos del usuario. Por esta razón, el razonamiento realizado debe incluir elementos fundamentales dentro de su análisis. 
En primer lugar, se debe considerar la calidad de vida de la persona como componente central para cualquier curso de acción a seguir. De igual manera, se debe promover la ocupación y participación del usuario, asumiendo que estos poseen un valor ético basado en los principios bioéticos, en las éticas del cuidado y virtudes, y en la dignidad de la persona. Por otro lado, se debe cambiar la mirada en torno a la muerte, promoviendo la ética del buen morir como una manera de enfrentar este proceso. Para todo lo anterior, es de gran relevancia recordar que nuestro quehacer se encuentra centrado en el usuario, por lo que cualquier decisión debe ir en coherencia con sus deseos, respetando y considerando su autodeterminación, siendo el diálogo la principal herramienta recomendada. La filosofía tanto de los cuidados paliativos como de la terapia ocupacional incluyen el sentido y significado para la persona, no solo de su ocupación y participación, sino que también del propio proceso de muerte y lo que esto conlleva.

Reconociendo la significancia que han adquirido los cuidados paliativos durante los últimos años en términos de reflexión y desarrollo, es importante mencionar las limitaciones encontradas en el proceso de recopilación de información para este ensayo. A nivel nacional no fueron encontrados autores que hablaran de la intervención de terapia ocupacional en cuidados paliativos, y aquellos que profundizan en lo paliativo, lo hacen desde otras profesiones del área de la salud. Es en revisiones internacionales donde se observa el mayor desarrollo teórico de este tema desde la terapia ocupacional. De esta manera, se deja la invitación a investigar y debatir en torno a un tema relevante y actual como éste.

\section{Agradecimientos}

A los docentes tanto de mi escuela como a aquellos docentes clínicos que compartieron sus conocimientos en las prácticas, por siempre instar a la reflexión teórica en pro del desarrollo de nuestra profesión, y al Dr. José Ignacio Vinay, por el apoyo, revisión y aportes realizados en este manuscrito.

\section{REFERENCIAS BIBLIOGRÁFICAS}

(1) MÉNDEZ, L. Historia de los Cuidados Paliativos. Módulo 1. Diplomado Cuidados Paliativos Oncológicos. Facultad de Medicina. Universidad de Chile. 2011.

(2) CORREGIDOR, A. Terapia ocupacional en cuidados paliativos: ocupar antes de morir. Revista Gallega de Terapia Ocupacional [Revista en internet]; 2010; monográfico 3; 185213.

(3) MINISTERIO DE SALUD DE CHILE. Guía Clínica Alivio del dolor por Cáncer Avanzado y Cuidados Paliativos.1 ${ }^{\text {a }}$ edición; Santiago; Minsal 2005.

(4) GÓMEZ, S. Primer módulo: Introducción a los modelos de práctica. Módulo Instruccional. Asignatura Modelos de Intervención en Salud Física. $2^{\text {a }}$ edición; Escuela de Terapia Ocupacional; Universidad de Chile; 2009; p. 9-11. 
(5) MATTINGLY, C. FLEMING, M. Clinical reasoning: Forms of inquiry in a therapeutic practice. F.A. Davis Philadelphia 1994

(6) SCHELL, B. Razonamiento Clínico: la base de la práctica. Willard \& Spackman: Terapia Ocupacional. 10ª edición; Editorial Panamericana; 2005; p. 131-139.

(7) LOLAS, F. Temas de Bioética. 2a edición; Editorial Universitaria; 2003.

(8) BARRANTES, M. RODRIGUEZ, E. y LAMA, A. Relación médico-paciente: derechos del adulto mayor. Acta Bioethica; 2009; 15(2): 216-221.

(9) BERRUETA, M. BELLIDO, J. y ARRASCO, L. Los principios éticos de la intervención en Terapia Ocupacional. Revista Gallega de Terapia Ocupacional; 2004; 1: 134.

(10) PESSINI, L. y BERTACHINI, L. Nuevas perspectivas en cuidados paliativos. Acta Bioethica; 2006; 12 (2): 231-242.

(11) GÓMEZ, M. ¿Morir en casa o en el hospital?. Módulo 1. Diplomado Cuidados Paliativos Oncológicos. Facultad de Medicina; Universidad de Chile; 2011.

(12) GÓMEZ, M. Modelos de asistencia en cuidados paliativos. Módulo 1. Diplomado Cuidados Paliativos Oncológicos. Facultad de Medicina; Universidad de Chile; 2011.

(13) VALLEJO, M. Rehabilitación y cuidado paliativo. Persona y Bioética; 2005; 9: 58-63.

(14) WRIGHT-ST CLAIR, V. Caring: the moral motivation for good occupational therapy practice. Australian Occupational Therapy Journal; 2001; 48: 187-199.

(15) LOLAS, F. Dilemas éticos: deber y virtud. Bioética y antropología médica. $2^{\mathrm{a}}$ edición; Editorial Mediterráneo; 2003; p. 132.

(16) CERECEDA, L. Ética de los cuidados paliativos. En: ESCRÍBAR, A. PÉREZ, M. y VILLARROEL, R. Bioética, fundamentos y dimensión práctica. $2^{\mathrm{a}}$ edición; Editorial Mediterráneo; 2008; p. 309-316.

(17) DIGE, M. Occupational therapy, professional development, and ethics. Scandinavian Journal of Occupational Therapy; 2009; 16: 88-98.

(18) TOWNSEND, E. y WILCOCK, A. Occupational Justice. Introduction to occupation. Upper Saddle River, NJ: Prentice-Hall; p. 243-273.

(19) LOLAS, F. Ética del buen morir. Escritos sobre vejez, envejecimiento y muerte. $1^{\mathrm{a}}$ edición; Editorial Campus; Universidad Arturo Prat; 2002; p. 255-260.

(20) DOMÍNGUEZ, O. Objeción de conciencia, la muerte y el morir en enfermedades en etapa terminal. Acta Bioethica; 2009, 15 (1): 94-99.

(21) PYRRHO, M. CORNELLI, G. y GARRAFA, V. Dignidad humana, reconocimiento y operalización del concepto. Acta Bioethica; 2009; 15 (1): 65-69.

(22) LOLAS, F. Las dimensiones bioéticas de la vejez. Acta Bioethica; 2001; 1: 57-70.

(23) ROMERO, D. y MORUNO, P. Razonamiento clínico y solución de problemas. Terapia Ocupacional, teoría y técnicas. 1ª edición; Editorial Masson; 2003; p. 297-314. 